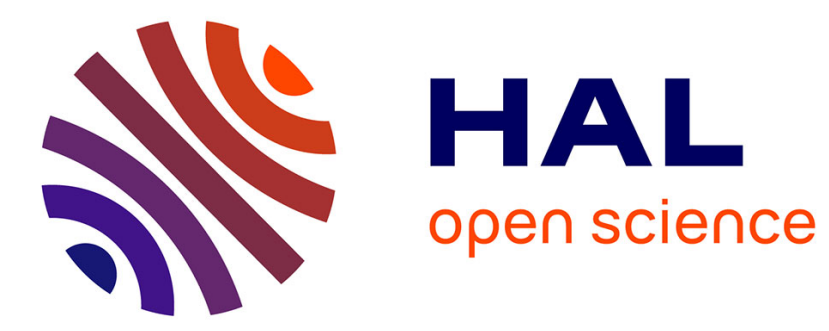

\title{
Maximum Likelihood Estimator For Doppler Parameter And Cramer Rao Bound In ZP-OFDM UWA Channel
}

\author{
Bastien Lyonnet, Cyrille Siclet, Jean-Marc Brossier
}

\section{To cite this version:}

Bastien Lyonnet, Cyrille Siclet, Jean-Marc Brossier. Maximum Likelihood Estimator For Doppler Parameter And Cramer Rao Bound In ZP-OFDM UWA Channel. ISCCSP 2008 - 4th International Symposium on Communications, Control and Signal Processing, May 2010, Limassol, Cyprus. pp.n.c. hal-00638172

\section{HAL Id: hal-00638172 \\ https://hal.science/hal-00638172}

Submitted on 4 Nov 2011

HAL is a multi-disciplinary open access archive for the deposit and dissemination of scientific research documents, whether they are published or not. The documents may come from teaching and research institutions in France or abroad, or from public or private research centers.
L'archive ouverte pluridisciplinaire HAL, est destinée au dépôt et à la diffusion de documents scientifiques de niveau recherche, publiés ou non, émanant des établissements d'enseignement et de recherche français ou étrangers, des laboratoires publics ou privés. 


\title{
Maximum Likelihood Estimator For Doppler Parameter And Cramer Rao Bound In ZP-OFDM UWA Channel
}

\author{
Bastien Lyonnet, Cyrille Siclet, Jean-Marc Brossier
}

\begin{abstract}
A Doppler estimation system using a maximum likelihood criterion is presented in the context of underwater acoustic communications between moving transmitter/receiver. We simulate the method for the estimation of the Doppler effect induced by an underwater acoustic channel (UWA) using Zero Padded-Orthogonal Frequency Division Multiplexing (ZPOFDM). Among the wide range of physical processes that impact OFDM communications through the underwater environment, Doppler effect is an important cause of loss of orthogonality and must be compensated before discrete Fourier transform based demodulation can be performed. The proposed approach is based on the perfect knowledge of the emitted signal during one block and rely on the assumptions of a small variation of the Doppler effect induced by the canal between two ZP-OFDM blocks and, above all, during one block.
\end{abstract}

\section{INTRODUCTION}

During the last three decades, underwater acoustic communications have received a growing interest. In the same time, OFDM modulation has been extensively developed in the context of wireless communication systems for its robustness against frequency selective channels. The idea comes naturally to use OFDM in underwater channel. But the ocean is a temporally and spatially varying propagation environment. The Doppler effect, induced by the channel, both affects the carrier tracking and the symbol synchronization. Relative motion between transmitter and receiver is usually unavoidable. This complicates the use of wide band, high speed acoustic communication techniques. The performance of a conventional OFDM receiver is severely limited by the intercarrier interference (ICI) due to the Doppler effect. Thus, several techniques have recently been developed in order to estimate and compensate it [1], [2].

In this paper, we focus on the receiver design. We developed a Doppler estimation method using a Maximum Likelihood (ML) criterion. This method relies on the perfect knowledge of the transmitted signal during one OFDM block. Considering communications between two underwater vehicles, we may realize the assumption that the Doppler will still be the same on the following blocks. Simulations are made using a channel with same Doppler effect on each path. Moreover, we also assume that the Doppler effect is constant from the beginning to the end of one OFDM block. Finally, measures of the estimator variance

This work was partly supported by the French ANR (Agence Nationale de la Recherche) LURGA project.

B. Lyonnet, C. Siclet and J.-M. Brossier are with GIPSA-lab, 961 rue de la Houille Blanche, BP46 38402 Grenoble Cedex, France bastien. lyonnet @gipsa-lab.grenoble-inp. fr cyrille.sicletagipsa-lab.grenoble-inp.fr jean-marc.brossier@gipsa-lab.grenoble-inp.fr are realized and compared to the Cramer Rao Bound (CRB).

\section{GENERAL CONTEXT}

The framework of our study is the transmission of digital information in shallow water environment. We first recall here the fundamentals of the multicarrier communication system we use, the OFDM with Zero Padding (ZP), and we describe then the channel model we have used.

\section{A. OFDM with Zero Padding}

The principle of ZP-OFDM is to use several carriers in parallel, $f_{m}\left(f_{m}=f_{0}+m F_{0}\right),-M / 2 \leq m \leq M / 2-$ 1 , with $M$ the number of carriers, with a symbol duration slightly higher than necessary. Indeed, since the frequency spacing $F_{0}$ is identical between each carrier frequency, then the minimum symbol duration is equal to $T_{0}=1 / F_{0}$. And yet, in ZP-OFDM, a symbol duration is in fact equal to $T=$ $T_{0}+T_{g}>T_{0}$. That is why $T_{g}$ is called a guard time. Then, for $-T_{g}-T_{0} / 2 \leq t \leq T_{0} / 2$ (one symbol duration), the ZP-OFDM baseband signal writes:

$$
s(t)=\sum_{m=-\frac{M}{2}}^{\frac{M}{2}-1} c[m] e^{j 2 \pi m F_{0} t} g(t)
$$

and the transmitted signal is

$$
s_{\mathrm{HF}}(t)=\Re\left\{s(t) e^{j 2 \pi f_{0} t}\right\}
$$

with $c[m]$ the transmitted data (QPSK ${ }^{1}$ symbols in here) and with $g(t)=1 / \sqrt{T_{0}}$ for $t \in\left[-T_{0} / 2, T_{0} / 2[\right.$, and $g(t)=0$ otherwise. Thus, $s(t)=0$ for $-T_{g}-T_{0} / 2 \leq t<-T_{0} / 2$, which results in a Zero Padding operation after sampling of the signal. It is worthwhile recalling that this causes a loss in spectral efficiency (no information is transmitted during this time interval), but it considerably simplifies the equalization procedure. If the guard interval is long enough, there will be no interference between OFDM symbol at the reception [3, chapter 11].

\section{B. Underwater acoustic channel model}

Our modelisation relies on several assumptions. First of all, we suppose that the acoustic channel can be described thanks to a time-varying multipath model. We moreover assume that the time-varying nature of the channel during one OFDM block is mainly caused by the relative motion between the receiver and the transmitter [1]. This assumption

${ }^{1}$ QPSK : Quaternary Phase Shift Keying 
is reasonable considering that the coherence time of an UWA channel is about one second [4] and a typical duration for $T$ is $200 \mathrm{~ms}$, depending on the number of subcarriers we choose.

Then, assuming a constant relative speed $v$ between the emitter and the receiver, and denoting $c$ the sound velocity and $\theta_{l}$ the arrival angle of path $l$, the received modulated signal $r_{\mathrm{HF}}(t)$ writes:

$$
r_{\mathrm{HF}}(t)=\sum_{l=1}^{L} A_{l} s_{\mathrm{HF}}\left(t-\frac{v \cos \left(\theta_{l}\right)}{c} t-\tau_{l}\right)+n_{\mathrm{HF}}(t),
$$

$L$ being the number of propagation paths, $A_{l}$ the amplitude of the $l$-th path and $\tau_{l}$ the corresponding path delay, and $b_{\mathrm{HF}}(t)$ being an additive white gaussian noise in the band of interest. What is more, if the receiver and the transmitter are sufficiently far away from each other, each propagation path seems to come from the same arrival angle, which means that $\theta_{l} \approx \theta_{1}$ for $1 \leq l \leq L$, so that, denoting $d=1-\frac{v \cos \left(\theta_{l}\right)}{c}$ :

$$
r_{\mathrm{HF}}(t)=\sum_{l=1}^{L} A_{l} s_{\mathrm{HF}}\left(d t-\tau_{l}\right)+n_{\mathrm{HF}}(t)
$$

Thus, using equations (2) and (4) we get:

$$
\begin{aligned}
r_{\mathrm{HF}}(t) & =\sum_{l=1}^{L} A_{l} \Re\left\{s\left(d t-\tau_{l}\right) e^{j 2 \pi f_{0}\left(d t-\tau_{l}\right)}\right\}+n_{\mathrm{HF}}(t) \\
& =\sum_{l=1}^{L} A_{l} \Re\left\{s\left(d t-\tau_{l}\right) e^{j 2 \pi f_{0}\left((d-1) t-\tau_{l}\right)} e^{j 2 \pi f_{0} t}\right\}+n_{\mathrm{HF}}(t)
\end{aligned}
$$

so that the lowpass version $r(t)$ of the received signal becomes:

$$
r(t)=\sum_{l=1}^{L} A_{l} s\left(d t-\tau_{l}\right) e^{j 2 \pi f_{0}\left((d-1) t-\tau_{l}\right)}+n(t)
$$

with $n(t)$ the equivalent lowpass gaussian noise.

Given that the intercarrier space is equal to $F_{0}$ and that we use $M$ carriers, the signal occupancy bandwidth is about $M F_{0}+B$, with $B$ the frequency occupancy bandwidth of $g(t)$ which is about $F_{0}$. Thus, for $M$ sufficiently high and discarding the first and last carriers, we can reduce the signal occupancy bandwidth to $W<M F_{0}$ so that the sampling period $T_{s}=\frac{1}{M F_{0}}$ respects the sampling theorem. So finally, after sampling at the instant $t_{k}=t_{0}+k T_{s}$, we have the signal :

$r[k]=\sum_{l=1}^{L} \alpha_{l} s\left(d k T_{s}+t_{0}-\tau_{l}\right) e^{j 2 \pi f_{0}(d-1)\left(k T_{s}+t_{0}\right)}+n[k]$

with $r[k]=r\left(t_{0}+k T_{s}\right), n[k]=n\left(t_{0}+k T_{s}\right)$, and $\alpha_{l}=$ $A_{l} e^{-2 \pi j f_{0} \tau_{l}}$, and $-\frac{T_{g}+\frac{T_{0}}{2}+t_{0}}{d T_{s}} \leq k<\frac{\frac{T_{0}}{2}-t_{0}}{d T_{s}}$. It is worthwhile noting that contrary to the radio-mobile case, the Doppler effect does not only causes a frequency shift but also a time compression/dilatation that can not be neglected. Indeed, in underwater acoustic communication, $c=1500 \mathrm{~m} / \mathrm{s}$ and the maximal speed is about $v_{\max }=6 \mathrm{~m} / \mathrm{s}$, which means that maximal Doppler value is about $d_{\max }=1 \pm 0.004$, whereas for radio-mobile communication we rather have $v_{\max }=$ $60 \mathrm{~m} / \mathrm{s}$ and $c=3.10^{8} \mathrm{~m} / \mathrm{s}$ so that $d_{\max }=1 \pm 2.10^{-7}$. This means that, during a 1024 symbols OFDM block, underwater acoustic Doppler causes a 4 samples shift, whereas radiomobile one causes a delay equal to $2 \cdot 10^{-4}$ times one sample duration.

\section{ML ESTIMATOR AND CRB}

In here, we restrict to the simplified case where $r[k]=$ $s\left(d k T_{s}\right) e^{j 2 \pi f_{0}(d-1) k T_{s}}+n[k]$. In other words, we consider only one path and we assume that the received signal has already been equalized and synchronized so that the only perturbation remaining is due to the gaussian noise and to the Doppler effect. Without loss of generality, we can assume that $t_{0}=-\left(T_{g}+\frac{T_{0}}{2}\right)$, and moreover, given that each block begins with a guard time constituted of a signal equal to zero, we can assume that $-T_{g}-\frac{T_{0}}{2} \leq t \leq \frac{T_{0}}{2}$ in spite of the dilatation/compression effect, by adjusting the guard time duration. So, assuming also that $T_{g}=M_{g} T_{s}$, we have $0 \leq k \leq M+M_{g}-1=K-1$.

$\begin{array}{cccc}\text { Let } & \text { us denote } \boldsymbol{R}= & {[r[0], \ldots, r[K-1]]^{T},} \\ N & = & {[n[0], \ldots, n[K-1]]^{T}} & \text { and } \boldsymbol{S}(d)\end{array}=$ $[s[0](d), \ldots, s[M-1](d)]^{T}$, with ${ }^{T}$ the transpose operator and $s[k](d)=s\left(d k T_{s}+t_{0}\right) e^{j 2 \pi f_{0}(d-1)\left(k T_{s}+t_{0}\right)}$. Thus, we have:

$$
\boldsymbol{R}=\boldsymbol{S}(d)+\boldsymbol{N}
$$

We assume that $\boldsymbol{S}(1)$ is known at the receiver side, so that we want to estimate $d$ with the knowledge of $\boldsymbol{R}$ and $\boldsymbol{S}(1)$. The likelihood of $d$ is therefore:

$$
\Lambda=f(\boldsymbol{R} / \boldsymbol{S}(1) ; d)=\frac{1}{\sqrt{2 \pi \sigma^{2}} K} e^{-\frac{1}{2 \sigma^{2}}(\boldsymbol{R}-\boldsymbol{S}(d))^{H}(\boldsymbol{R}-\boldsymbol{S}(d))}
$$

given that the components of $\boldsymbol{N}$ are gaussian, independent, zero-mean and with variance $\sigma^{2}$, with ${ }^{H}$ the transpose conjugate operator and $K=M+M_{g}$. Then, the best estimation of $d$ is:

$$
\hat{d}=\operatorname{argmax}_{d}\{f(\boldsymbol{R} / \boldsymbol{S}(1) ; d)\}
$$

Then, under the assumption that the maximum likelihood estimator is unbiased $(E[\hat{d}-d]=0)$, its Cramer Rao bound is given by $[5, \mathrm{pp}$ 65-72].

$$
\mathrm{CRB}=\frac{1}{-\mathrm{E}\left[\frac{\partial^{2}}{\partial d^{2}} \ln \Lambda\right]}
$$

Using (10), we get:

$$
\ln \Lambda=-\frac{K}{2} \ln \left(2 \pi \sigma^{2}\right)-\frac{1}{2 \sigma^{2}}(\boldsymbol{R}-\boldsymbol{S}(d))^{H}(\boldsymbol{R}-\boldsymbol{S}(d))
$$

and

$$
\begin{aligned}
& \frac{\partial}{\partial d} \ln \Lambda=-\frac{1}{2 \sigma^{2}} \frac{\partial}{\partial d}\left\{\boldsymbol{R}^{H} \boldsymbol{R}-\boldsymbol{S}(d)^{H} \boldsymbol{R}-\boldsymbol{R}^{H} \boldsymbol{S}(d)\right. \\
& \left.+\boldsymbol{S}(d)^{H} \boldsymbol{S}(d)\right\} \\
& \approx-\frac{1}{2 \sigma^{2}} \frac{\partial}{\partial d}\left\{-\boldsymbol{S}(d)^{H} \boldsymbol{R}-\boldsymbol{R}^{H} \boldsymbol{S}(d)+\boldsymbol{S}(d)^{H} \boldsymbol{S}(d)\right\}
\end{aligned}
$$


for $K$ sufficienlty high. Indeed, $\boldsymbol{R}^{H} \boldsymbol{R}$ is equal to the received energy of the signal during one OFDM block, which is approximately constant for $K$ sufficiently high, and thus independent from $d$. Then,

$$
\begin{aligned}
& \frac{\partial^{2}}{\partial d^{2}} \ln \Lambda=\frac{1}{2 \sigma^{2}}\left\{\frac{\partial^{2}}{\partial d^{2}} \boldsymbol{S}(d)^{H} \boldsymbol{R}+\boldsymbol{R}^{H} \frac{\partial^{2}}{\partial d^{2}} \boldsymbol{S}(d)\right. \\
&- \frac{\partial^{2}}{\partial d^{2}} \boldsymbol{S}(d)^{H} \boldsymbol{S}(d)-\frac{\partial}{\partial d} \boldsymbol{S}(d)^{H} \frac{\partial}{\partial d} \boldsymbol{S}(d) \\
&-\left.\frac{\partial}{\partial d} \boldsymbol{S}(d)^{H} \frac{\partial}{\partial d} \boldsymbol{S}(d)-\boldsymbol{S}(d)^{H} \frac{\partial^{2}}{\partial d^{2}} \boldsymbol{S}(d)\right\} \\
&= \frac{1}{2 \sigma^{2}}\left\{\frac{\partial^{2}}{\partial d^{2}} \boldsymbol{S}(d)^{H}(\boldsymbol{R}-\boldsymbol{S}(d))+\right. \\
&\left.(\boldsymbol{R}-\boldsymbol{S}(d))^{H} \frac{\partial^{2}}{\partial d^{2}} \boldsymbol{S}(d)-2 \frac{\partial}{\partial d} \boldsymbol{S}(d)^{H} \frac{\partial}{\partial d} \boldsymbol{S}(d)\right\}
\end{aligned}
$$

so that, with $\mathrm{E}\{\boldsymbol{R}-\boldsymbol{S}(d)\}=\mathrm{E}\{\boldsymbol{B}\}=0$ and using (12):

$$
\begin{aligned}
\mathrm{CRB} & =\frac{\sigma^{2}}{\frac{\partial}{\partial d} \boldsymbol{S}(d)^{H} \frac{\partial}{\partial d} \boldsymbol{S}(d)} \\
& =\frac{\sigma^{2}}{\sum_{k=0}^{K-1}\left|\frac{\partial s[k](d)}{\partial d}\right|^{2}}
\end{aligned}
$$

Let us recall that $s[k](d)=s\left(d k T_{s}+t_{0}\right) e^{j 2 \pi f_{0}(d-1)\left(k T_{s}+t_{0}\right)}$. Therefore, according to (1):

$$
\begin{gathered}
\frac{\partial s[k](d)}{\partial d}=\frac{\partial s\left(d k T_{s}+t_{0}\right)}{\partial d} e^{j 2 \pi f_{0}(d-1)\left(k T_{s}+t_{0}\right)}+ \\
j 2 \pi f_{0}\left(k T_{s}+t_{0}\right) s\left(d k T_{s}+t_{0}\right) e^{j 2 \pi f_{0}(d-1)\left(k T_{s}+t_{0}\right)} \\
=j 2 \pi\left(k T_{s}+t_{0}\right) e^{j 2 \pi f_{0}(d-1)\left(k T_{s}+t_{0}\right)}\left(f_{0} s\left(d k T_{s}+t_{0}\right)+\right. \\
\left.\quad \sum_{m=-\frac{M}{2}}^{\frac{M}{2}-1} m F_{0} c[m] e^{j 2 \pi m F_{0} d\left(k T_{s}+t_{0}\right)} g\left(d k T_{s}+t_{0}\right)\right)
\end{gathered}
$$

and

$$
\begin{aligned}
& \left|\frac{\partial s[k](d)}{\partial d}\right|^{2}=4 \pi^{2}\left(k T_{s}+t_{0}\right)^{2} \mid f_{0} s\left(d k T_{s}+t_{0}\right)+ \\
& \left.\quad \sum_{m=-\frac{M}{2}}^{\frac{M}{2}-1} m F_{0} c[m] e^{j 2 \pi m F_{0} d\left(k T_{s}+t_{0}\right)} g\left(d k T_{s}+t_{0}\right)\right|^{2}
\end{aligned}
$$

It is worthwhile noting that even if $\left|\frac{\partial s[k](d)}{\partial d}\right|^{2}$ depends upon the initial sampling instant $t_{0}$, the CRB is in fact independent of $t_{0}$ since it is computed thanks to a sum over $k$ and the set of values $k T_{s}+t_{0}$, which is in fact independent of $t_{0}$. We also underline that $g(t)$ is constant equal to $\frac{1}{\sqrt{T_{0}}}$ or to 0 so that its derivative is equal to 0 .

This bound depends on the complex signal $S$ derived according to the continuous real parameter $d$. It is worthwhile noting that the higher is the number of samples, the lower is the CRB.

\section{SIMULATION}

The emission of a ZP-OFDM symbol through the channel presented before is simulated. The UWA channel is doubly dispersive. The coherence time is close to the second, while the typical delay observed in shallow water environment is around $40 \mathrm{~ms}$ for a distance between source and receiver close to the kilometer. A study of the relation between capacity, distance and frequency as been done in [6]. Regarding the typical bandwidth in underwater acoustic communication, we choose a carrier frequency $f_{0}=20$ $\mathrm{kHz}, T=200 \mathrm{~ms}$ and the guard interval has a duration $T_{g}=53.5 \mathrm{~ms}$. We choose to simulate a communication over 1500 subcarriers. the guard interval is composed of 548 samples, so we have $K=2048$. The subcarrier spacing is $6.8 \mathrm{~Hz}$. QPSK modulation is used. We chose these values regarding those used in [1].

Typical transmission will concern underwater vehicles moving around 6 to 12 knots, this corresponds approximately to a speed between $3 \mathrm{~m} / \mathrm{s}$ and $6 \mathrm{~m} / \mathrm{s}$ and a Doppler parameter of $d=1 \pm 0.002$ and $d=1 \pm 0.004$, depending on the movement direction. The Doppler shift will be between 40 and $80 \mathrm{~Hz}$. A compensation is necessary to mitigate the Doppler effect. In order to illustrate the effect of the Doppler on the OFDM signal, we have realized several simulations illustrated in the figure (1). In these simulations, the number of subcarriers and the Doppler parameter is growing and affect the Binary Error Rate. Theses simulations allow us to determine from which value of the Doppler the information carrier by the OFDM signal is destroyed. For example here, with the configuration of $M=1500$ and $f_{0}=20 \mathrm{kHz}$, the $B E R$ is up to $10^{-2}$ for a Doppler parameter of $1+6.10^{-5}$. Considering this information, the desired estimator should have a standard deviation inferior to $2.10^{-5}$ in order to guarantee the decoding of the information after demodulation with a limited error rate.

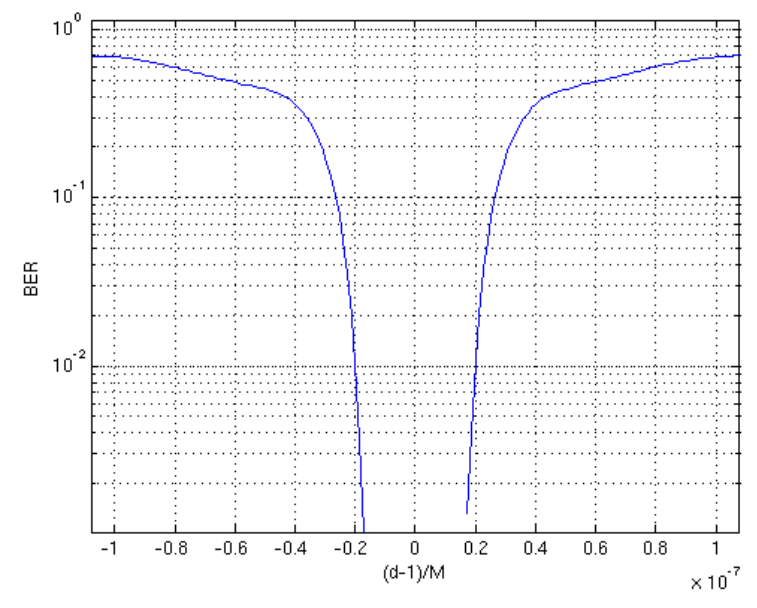

Fig. 1. Binary Error Rate for different Doppler parameter without any Doppler compensation.

At the receiver, we treat the sampled passband signal. First of all, a quick estimation of the Doppler parameter is realized using the method developed in [2]. This method is based on a measure of the dilatation/compression effect due 
to the Doppler effect. Then, an estimation of the Doppler parameter using the maximum likelihood estimator is done. The knowledge of the emitted signal is necessary for this part. The research of the maximum is realized using the Nelder-Mead Simplex method [3]. The compensation of the Doppler effect is based on the same idea as used in [1]. A rescaling of the signal is performed to compensate the influence of the Doppler on the signal $r$. Then, the residual offset is also compensated using null subcarriers [7]. With these two operations, the Doppler effect is completely compensated.

In order to test the estimator, we simulated two kinds of channels. In channel 1 , we only implemented Doppler and additive white gaussian noise. We receive the signal $r[k]=s\left(d k T_{s}+t_{0}\right) e^{j 2 \pi f_{0}(d-1)\left(k T_{s}+t_{0}\right)}+b[k]$. We have tested it with several values for the Doppler parameter $d$ and we show the Likelihood function for a value of $d=0.997$ in the figure (2). The maximum is clearly identified even with strong noise (SNR equal to $0 \mathrm{~dB}$ ). Channel 2 is a three paths channel with coefficient $A_{1}=0.6, A_{2}=0.1$ and $A_{3}=0.3$ and delays $\tau_{1}=0, \tau_{2}=10 \mathrm{~ms}$ and $\tau_{3}=40 \mathrm{~ms}$. But, during our estimation of $d$, we don't know these values. In fact, we still consider the model of $s[k](d)$ unchanged, $s[k](d)=s\left(d k T_{s}+t_{0}\right) e^{j 2 \pi f_{0}(d-1)\left(k T_{s}+t_{0}\right)}$. The likelihood function is shown in the figure (2). The maximum is still accurate. But the theoretical computation of the CRB is not valid anymore.

To illustrate our words, we show in figure (3) the variance of the estimator in the two channels and the theoretical Cramer Rao Bound. In order to fully understand these results, it is necessary to follow the demonstration made on the figure (1). The threshold value that the variance should not exceed is $4.10^{-10}$, corresponding to a standard deviation of $2.10^{-5}$. Looking at the figure (3), the CRB is below the threshold for a SNR inferior to $6.10^{-3}$. In theory, it is not possible to obtain an estimation of the Doppler parameter precise enough to recover the information of our OFDM signal with an SNR up to $-22 \mathrm{~dB}$. This limited SNR value will change with other signals and with the Doppler parameter. The variance of our ML estimator falls below the threshold for an SNR up to $1.6 \times 10^{-2}$. The results, with and without multipaths, are close.

Between a SNR of $10^{-2}$ and $10^{-1}$, the variance of the estimator follows the Cramer Rao Bound. Up to $10^{-1}$, the estimator is limited by the algorithm of the simplex search method.

We can compare our results with those of [1] and [2], using block Doppler estimation. In these articles, the precision of the estimator directly depends on the sampling period. It is shown that the resolution of their estimator is $1 / B T$ with $B$ the bandwidth of the signal. In our case, with a bandwidth of $10.2 \mathrm{kHz}$, the minimum variance of the estimator proposed in [2], will be $4.48 \times 10^{-7}$. As we have said it before, it is not precise enough for our computation. But, if we compare the ML estimator with the one used in [1] for the same number of samples, the ML estimator seems to be more robust and more precise.

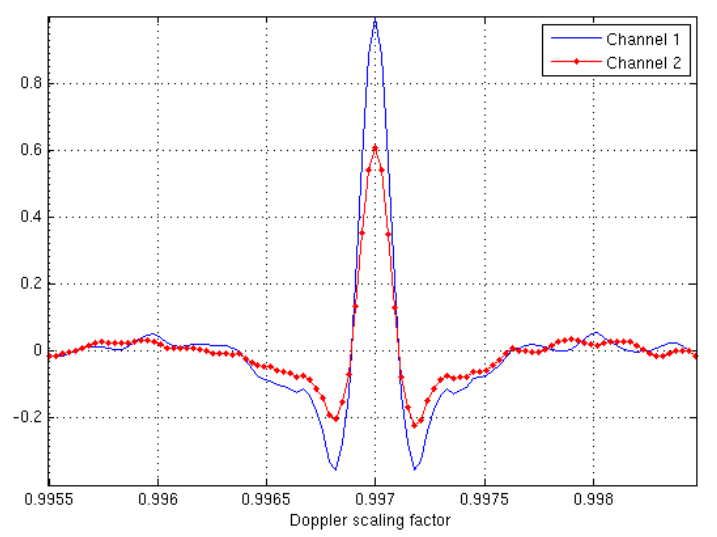

Fig. 2. Maximum Likelihood as a function of the parameter $\hat{d}, d=0.997$, $\mathrm{SNR}=0 \mathrm{~dB}$

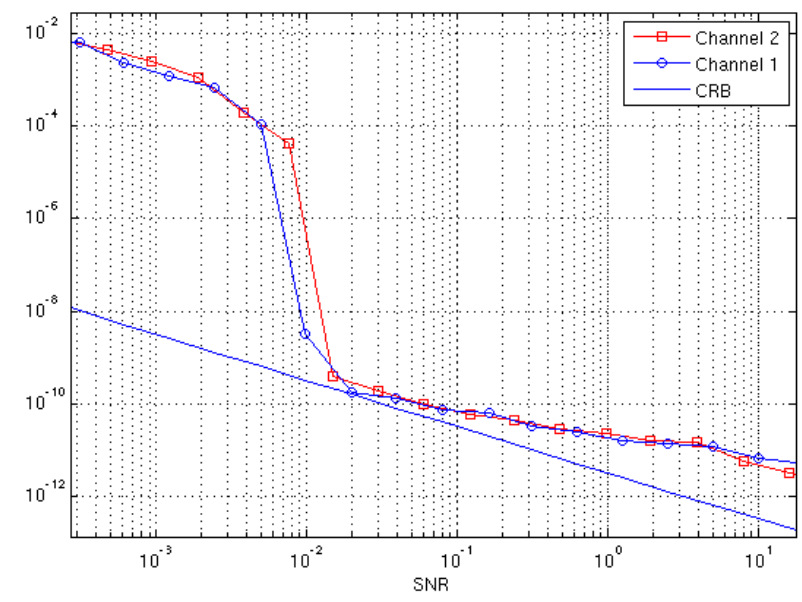

Fig. 3. Cramer Rao Bound and Variance of the estimator as a function of the Signal to noise ratio $d=0.997$.

\section{CONCLUSION}

In this paper, we have investigated the application of an estimator of the Doppler shift and the Doppler distortion using a Maximum Likelihood criterion applied to ZP-OFDM in UWA channel. The receiver is based on block-by-block processing. Estimation needs the perfect knowledge of the transmitted signal during one OFDM block. We have to assume that the Doppler parameter during the following block will not change and the previous Doppler estimated will still be correct. The method proposed is tested in simulations for a channel with and without multiple paths. The estimation is correct in both cases. Simulations show the variance of the estimator compared to Cramer Rao Bound. Further improvements will focus on an estimator using partial knowledge of the signal allowing an estimation of the Doppler parameter during each block and communication in the same time. 


\section{REFERENCES}

[1] Baosheng Li, Shengli Zhou, M. Stojanovic, L. Freitag, and P. Willett, "Non-uniform doppler compensation for zero-padded OFDM over fastvarying underwater acoustic channels," in OCEANS 2007 - Europe, June 2007, pp. 1-6.

[2] B.S. Sharif, J. Neasham, O.R. Hinton, and A.E. Adams, "A computationally efficient doppler compensation system for underwater acoustic communications," Oceanic Engineering, IEEE Journal of, vol. 25, no. 1, pp. 52-61, Jan 2000.

[3] J. Proakis and M. Salehi, Digital Communications, McGraw-Hill International Edition, 2008.
[4] James Preisig, "Acoustic propagation considerations for underwater acoustic communications network development," SIGMOBILE Mob. Comput. Commun. Rev., vol. 11, no. 4, pp. 2-10, 2007.

[5] HL Van Trees, Detection, estimation, and modulation theory part I, vol. 1, McGraw-Hill International Edition, 1968.

[6] Milica Stojanovic, "On the relationship between capacity and distance in an underwater acoustic communication channel," SIGMOBILE Mob. Comput. Commun. Rev., vol. 11, no. 4, pp. 34-43, 2007.

[7] H. Liu and U. Tureli, "A high-efficiency carrier estimator for OFDM communications," Communications Letters, IEEE, vol. 2, no. 4, pp. 104-106, Apr 1998. 